\title{
Experimental analysis and modelling of the strain-rate sensitivity of sheet niobium
}

\author{
Lorenzo Peroni ${ }^{1, *}$, Martina Scapin ${ }^{1}$ \\ ${ }^{1}$ Politecnico di Torino, Department of Mechanical and Aerospace Engineering, Corso Duca degli Abruzzi 24, 10129 Turin, Italy
}

\begin{abstract}
The niobium is currently used for the construction of the superconducting radio frequency (RF) Crab Cavity for the particle accelerator LHC at CERN in Geneva. An alternative technique to traditional forming methods is the electrohydraulic forming (EHF), in which ultrahigh-speed deformation of blank sheets is performed by using shockwaves electrically induced in water. A big effort is made for the analysis of the forming processes by FEM simulations, which require the definition of an appropriate flow stress material model. With this aim, in the present work, a testing campaign was performed in tension on sheet specimens with a rectangular cross-section at different strain-rates, up to $10^{3} \mathrm{~s}^{-1}$. The obtained results showed the material is strongly sensitive to strain-rate, as expected for a pure BCC metal. The data, were processed via a reverse engineering procedure, based on finite element simulations of the experimental tests. This methodology allowed the identification of a tabular flow stress model (MAT_224 implemented in LSDYNA) for the prediction of the material behaviour as a function of the plastic strain, strain-rate and temperature.
\end{abstract}

\section{Introduction}

The niobium is currently used for the construction of the superconducting radio frequency (RF) Crab Cavity developed in the framework of the High-Luminosity Large Hadron Collider (HL-LHC) at CERN in Geneva [1]. Crab cavities are special cavities which produce rotation of the proton beam leading to a larger number of collisions by increasing the area of intersection between the two crossing beams [2]. The ability of the cavity to rotate the protons beam with the right frequency strongly depends on its shape, hence tight geometrical tolerances are required. These considerations explain the interest in the fabrication method.

Superconducting cavities are traditionally formed from superconducting material sheets, followed by trim machining and electron-beam welding. An alternative technique to traditional shaping methods, such as deepdrawing and spinning, is electrohydraulic forming (EHF). In EHF, half-cells are obtained through ultrahigh-speed deformation of blank sheets, by using shockwaves induced in water by a pulsed electrical discharge. With respect to traditional methods, such a highly dynamic process can yield interesting results in terms of effectiveness, repeatability, final shape precision, higher formability, and reduced springback. During this process, the component is produced with speeds in the order of $100 \mathrm{~m} / \mathrm{s}$.

As mentioned before, a big effort is made for the analysis of the forming processes by FEM simulations, which allowed a faster iteration on the design of the fabrication tool, a better understanding of the involved phenomena in the process, the steering of the manufacturing choices and, ultimately, an estimation of the expected shape outcome and the thickness distribution.

As expected for a pure BCC metal, niobium should be strongly sensitive to strain-rate: hence the quality and reliability of the FE results strongly depend by the definition of an appropriate strain-rate dependent flow stress material model. With this aim, in the present work, a testing campaign was performed in tension on sheet specimens with a rectangular cross-section at different strain-rates from static to highly dynamic loading conditions. In more detail, the tensile behaviour was investigated at 4 different strain-rates: quasi-static (nominally at $10^{-3} \mathrm{~s}^{-1}$ ), medium (nominally at $10^{-1} \mathrm{~s}^{-1}$ and $10^{1} \mathrm{~s}^{-1}$ ) and high strain-rate (nominally at $10^{3} \mathrm{~s}^{-1}$ ). The last condition was reached by means of a Hopkinson Bar setup in direct configuration properly developed for sheet specimens. All the tests were recorded by using high-resolution or high-speed cameras, depending on the strain-rate of the test, in order to measure the gage length deformation. The experimental data were used in a reverse engineering approach to calibrate a proper strength model for the material behaviour prediction.

\section{Experimental setup and tests}

The specimens used in the testing campaign were obtained from pure niobium sheet and the sketch is reported in fig. 1: they have a rectangular gage crosssection of $4 \times 3.6 \mathrm{~mm}$ and a gage length of $5 \mathrm{~mm}$. The specimen fabrication was obtained by waterjet cutting in

Corresponding author: martina.scapin@polito.it 
order to reduce the effects of the increase of temperature during machining: as a matter of fact, also a high sensitivity to temperature was expected, hence it is necessary to limit the temperature increase to be sure to not modify the material microstructure or induce changes in the material behaviour before testing.
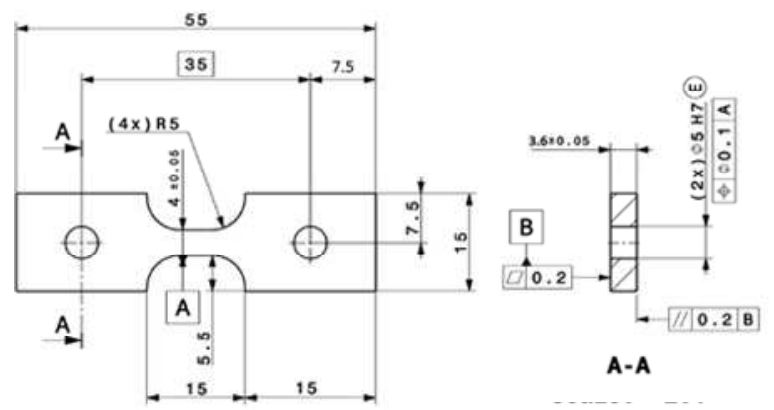

Fig. 1. Sketch of the specimen used in the testing campaign.

The tests were performed at four different strainrates. The quasi-static tests, nominally at $10^{-3} \mathrm{~s}^{-1}$, and the medium-low strain-rate tests, nominally at $10^{-1} \mathrm{~s}^{-1}$, were performed on a standard electro-mechanical testing machine (Zwick Z-100) by imposing a testing speed of $0.005 \mathrm{~mm} / \mathrm{s}$ and $0.5 \mathrm{~mm} / \mathrm{s}$, respectively. The medium strain-rate tests, nominally at $10^{1} \mathrm{~s}^{-1}$ were performed on a servo-hydraulic testing machine (Dartec HA 100), by imposing a testing speed of $50 \mathrm{~mm} / \mathrm{s}$. Finally, the high strain-rate tests, nominally at $10^{3} \mathrm{~s}^{-1}$, were performed on a Tension Hopkinson Bar setup in direct configuration, which was ad-hoc developed to test flat specimens. The operating principle of the direct setup for tensile tests is reported in [3].

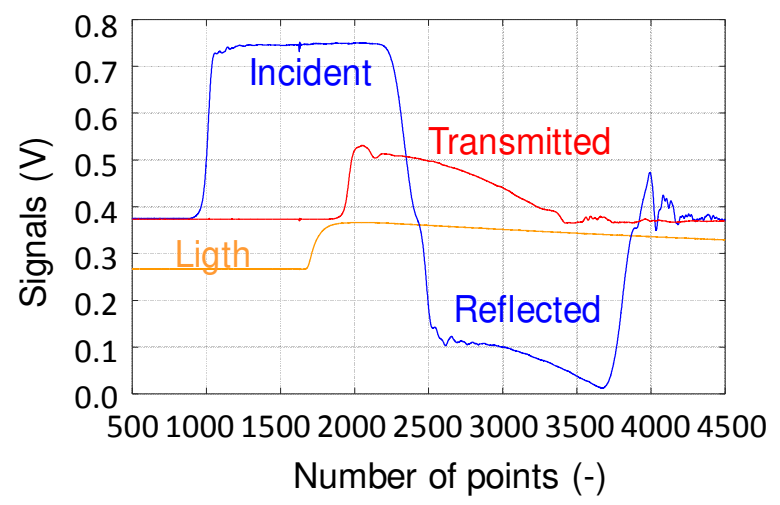

Fig. 2. Acquired signals at $2.5 \mathrm{MHz}$ : strain-gage signals on input and output bars and light sensor signal.

The setup consists of an accelerating gun of $2000 \mathrm{~mm}$, in which a hollow striker of $750 \mathrm{~mm}$ in length, made of glass-reinforced nylon, is launched by compressed air. The input and output bars are made of Ergal 7075-T6 and has a diameter of $16 \mathrm{~mm}$ and a length of $3000 \mathrm{~mm}$. The input bar is jointed with another bar made of martensitic steel 17-4PH with a diameter of $10 \mathrm{~mm}$ and a length of $3400 \mathrm{~mm}$. A hammer is mounted at the end of this bar and it is impacted by the striker to generate the tensile wave. The signals which travel the input and the output bars are measured by semiconductor strain-gages. An example of the acquired signals on input and output bars is reported in fig. 2 .

All the testing setups were equipped with a C-shape ends gripping system in which the specimen was fixed by pins (fig. 3). Different solutions were evaluated, but this was the best one especially for high strain-rate tests, in which the limiting of the inertia contribution induced by the gripping system is of fundamental importance.

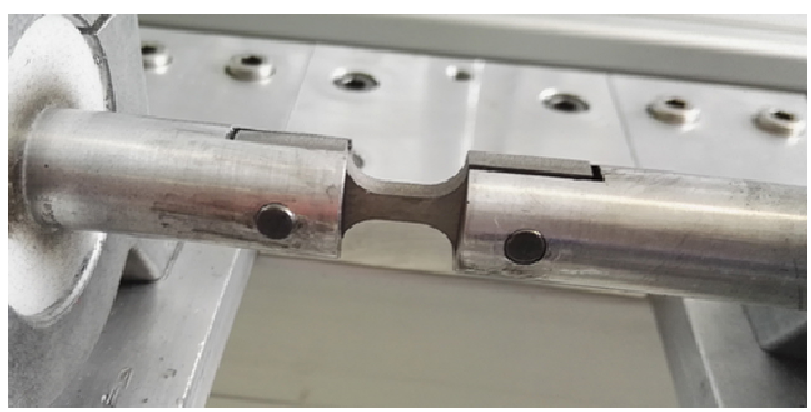

Fig. 3. Detail of the fixing system adopted on the Hopkinson Bar setup.
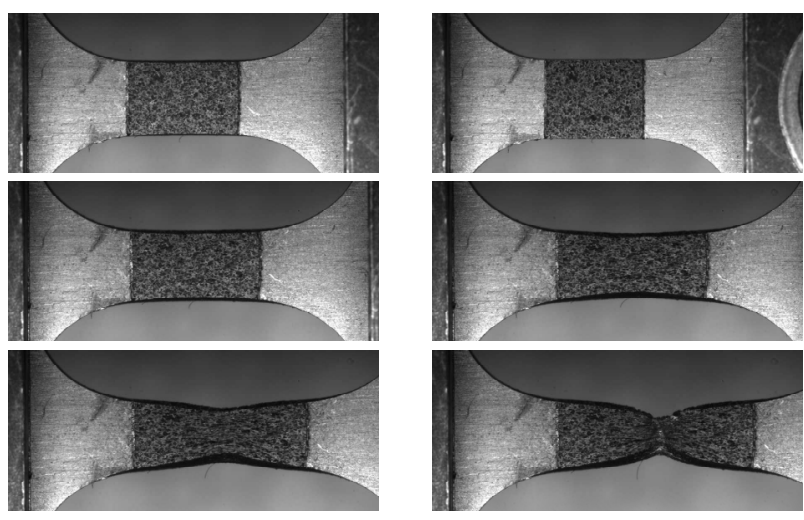

Fig. 4. Sequence of images of the deformation for a quasi-static test, nominally at $10^{-3} \mathrm{~s}^{-1}$ : images acquired at $0.5 \mathrm{fps}$ with a resolution of $2500 \times 2000 \mathrm{px}$ (the time interval between two subsequent images is $96 \mathrm{~s}$ ).

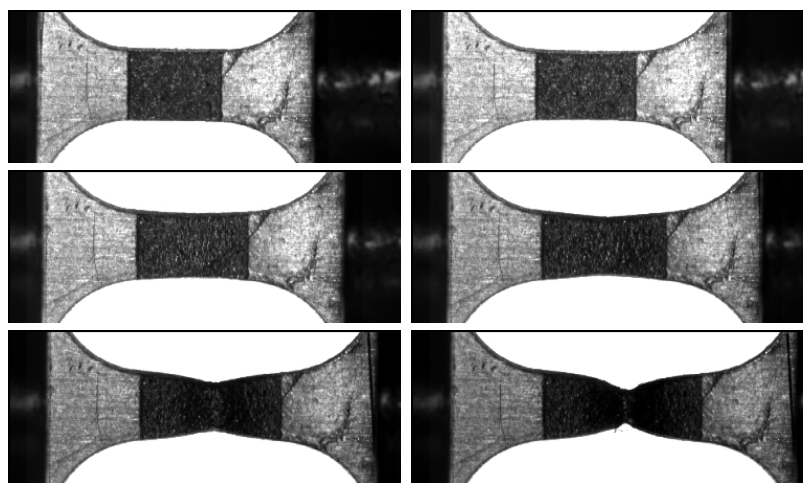

Fig. 5. Sequence of images of the deformation for a high strain-rate test, nominally at $10^{3} \mathrm{~s}^{-1}$ : video acquired at $100000 \mathrm{fps}$ with a resolution of $448 \times 144 \mathrm{px}$ (the time interval between two subsequent images is $90 \mu \mathrm{s}$ ).

In each case, the force was measured directly from the testing equipment, by means of load cells (straingage or piezoelectric cells) or semiconductor straingages. For each test, the specimen deformation in the 
gage length was measured by digital image elaboration of the videos recorded during the test. A speckle was performed on the gage length of each specimen: this allowed to be able to track the position of some points as well as the ends of the gage length. The optical extensometer technique was adopted in order to be able to more precisely evaluate the deformation of the gage length and to reduce the errors and the corrections needed if the stroke imposed by the machine was used. As a matter of fact, the last includes also the contributions of clearances and compliances of the entire measuring chain and the specimen heads.

By depending on the strain-rate a high-resolution or high-speed camera was used. For quasi-static and low strain-rate tests, the high-resolution camera Pixelink PLB777U was adopted with a framerate of $0.5 \mathrm{fps}$ and $50 \mathrm{fps}$, respectively. For medium and high strain-rate tests, the high-speed camera Photron SA5 was used with a framerate of $5000 \mathrm{fps}$ and $100000 \mathrm{fps}$, respectively. Of course, the videos were synchronized with the force. In order to be able to record the test, the image acquisition system was coupled with an appropriate lightening system able to generate the required level of light in relation to the sensor sensitivity and opening. For the high-resolution camera, a LED lightening system was used; for the high-speed camera, a pulsed light system was adopted and synchronized with the setup. For the Hopkinson Bar setup, the high-speed camera and the lightening system were synchronized by using the trigger generated by the incident wave signal on the input bar and by adding programmable delay, with a precision of about $10 \mu \mathrm{s}$. Two sequences of images taken from the recorded videos in case of quasi-static and high strainrate tests are reported in figs. 4 and 5.

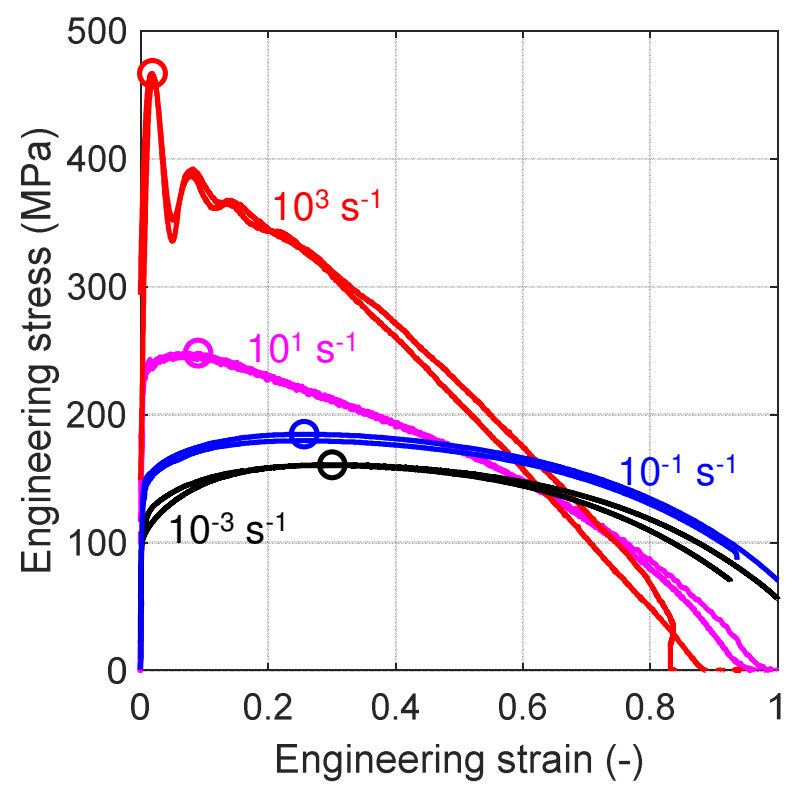

Fig. 6. Experimental results in terms of engineering stressstrain curves for all the loading conditions.

\section{Experimental results}

In fig. 6, the experimental results in terms of engineering stress-strain curves are reported. The data were obtained starting from force vs. displacement curve by referring to initial (nominal) specimen dimensions. For each loading condition, at least two repetitions are shown in order to check the repeatability of the material response: by comparing the results it is possible to conclude that the data scatter is low (fig. 6). From the diagram, it is also possible to notice that the material, as expected for a BCC pure metal, is strongly dependent on strain-rate: 6 orders of magnitude of increasing strainrate produce a consequently increase of about $300 \mathrm{MPa}$ in the yielding stress. The other important consequence of the strain-rate increment is the modification of the instability onset: higher the strain-rate, lower the deformation at instability. In particular, in dynamic regime, almost the whole curve is in post-necking regime, since the necking starts just after yielding. Therefore, the curves are mainly strictly decreasing. Another contribution which can be considered to start to be significant from medium/high strain-rate tests is the effect of the adiabatic heating due to the conversion of the stored plastic deformation: the influence is greater at high strain values and, if not balanced by the increasing of strain-rate in the necking region, could produce a further reduction of the mechanical response.

\section{Data analysis}

The best result, when a strength model identification is performed, is to obtain a unique formulation with the smallest set of parameters able to predict the material response as better as possible in several loading conditions (e.g. at least in strain, strain-rate and temperature). In order to fully meet this objective, it could be necessary to find a perfect correlation between the experimental data and the model. Often, simple strength models, for which the parameters identification is quite easy and requires a limited number of tests to be performed, are not able to correctly predict a real material response, especially over wide ranges of variation of the involved variables. In this case, a possible solution could be to define some specific paths on the (hyper)surface which defines the stress as a function of all the involved variables. The meaning of each path is the definition of the material response in a particular loading condition on which it was possible to obtain a good matching between the chosen material model and the experimental data. This is equivalent to a local approach, since usually the range of variation of the involved variables in a single test is present but is limited with comparison to the entire ranges investigated in a complete testing campaign. As a matter of fact, due to necking the strain-rate can be increased by a factor 10 , while for example the actual testing campaign investigated the strain-rate effects over 6 orders of magnitude.

This is the idea at the basis of the analysis performed to obtain a flow stress law from niobium data: the effects 
of strain-rate and temperature on the material response are so significant that simple strength models, such as Johnson-Cook or Zerilli-Armstrong ones, could not able to predict the material behaviour with a sufficient level of accuracy in all the loading conditions. Hence, the authors proposed the use of a tabular model defined by a series of plastic (isothermal) flow stress-strain curves at different loading conditions in terms of strain-rate and temperature dependences. Each curve was obtained via a reverse engineering approach based on FE simulations of all the investigated loading conditions in a Multi-Case Optimization, MCO.

\subsection{Multi-Case Optimization}

The requirement of a reverse engineering approach came from the extension of the necking region and the need to obtain a model able to reproduce the material behaviour up to failure or up to high deformation level. Moreover, this approach can consider for the coupling between strain, strain-rate and temperature as well as the increasing of triaxiality after necking onset. A technique based on the necking profile (e.g. radial displacement of the specimen surface) could not be applied since the specimen is not axisymmetric, hence a more conventional approach, based on the curve matching in terms of macroscopic quantities, such as the force vs. stroke curves, was adopted. In this way, the necking profile is not taken into account as objective function in the optimization procedure, but it can be checked at the end of the procedure.

The FE simulations were performed by using the explicit non-linear code LS-DYNA and the optimization was managed by the software LS-OPT.

The FE model was made by 3D solids elements and represented an eighth of the specimen, obtained by imposing proper symmetry constraints. The experimental displacement was imposed to the nodes of the specimen head. The displacement was measured at the end of the gage length, in accordance with the procedure used in the experimental data elaboration and, finally, the force was measured in a plane perpendicular to the longitudinal direction.

The chosen material model was the MAT_224 implemented in LS-DYNA [4]. It is a table-defined elasto-viscoplastic model, in which arbitrary stress-strain curves can be defined at different strain-rates and temperatures, in accordance with the formulation:

$$
\sigma_{y}=k_{1}\left(\varepsilon_{p}, \dot{\varepsilon_{p}}\right) \frac{k_{t}\left(\varepsilon_{p}, T\right)}{k_{t}\left(\varepsilon_{p}, T_{R}\right)}
$$

where $k_{1}$ is a table in which one equivalent stress vs. effective plastic strain curve is defined for each strainrate and $k_{t}$ is a table in which one equivalent stress vs. effective plastic strain curve is defined for the reference temperature $T_{r}$ and for each other temperature. Each curve reported in the table $k_{1}$ represents the isothermal stress-strain curve at that rate. Between two subsequent curves, the interpolation is performed logarithmically in strain-rate. The internal heating due to plastic work was taken into account by means of Taylor-Quinney coefficient, which determines the quantity of plastic work converted into heat and it was fixed to 0.9.

In more details, four curves were defined at four different strain-rates in order to cover the entire range investigated by the experimental tests. Since the model does not extrapolate, the lowest curve was defined for a strain-rate $\left(3 \times 10^{-4} \mathrm{~s}^{-1}\right)$ lower than the minimum nominal one. Similarly, the highest curve was associated to a strain-rate $\left(5 \times 10^{3} \mathrm{~s}^{-1}\right)$ higher than the maximum nominal one to take into account the increase in strain-rate during necking. Each stress-strain curve (identified by the subscript $i$ ) was described by three parameters in accordance with the formulation of the hardening part of the Johnson-Cook model:

$$
\sigma_{y, i}=A_{i}+B_{i} \varepsilon_{p}^{n_{i}}
$$

The optimization was based on the minimization of the Mean Square Error between the target curve (i.e. experimental force vs. displacement curve) and the corresponding computed one. The objective was to identify all the sets of parameters, each of one related to a specific strain-rate. Since, as discussed before, high strain-rate tests are adiabatic, it is necessary to define also the thermal softening part of the model.

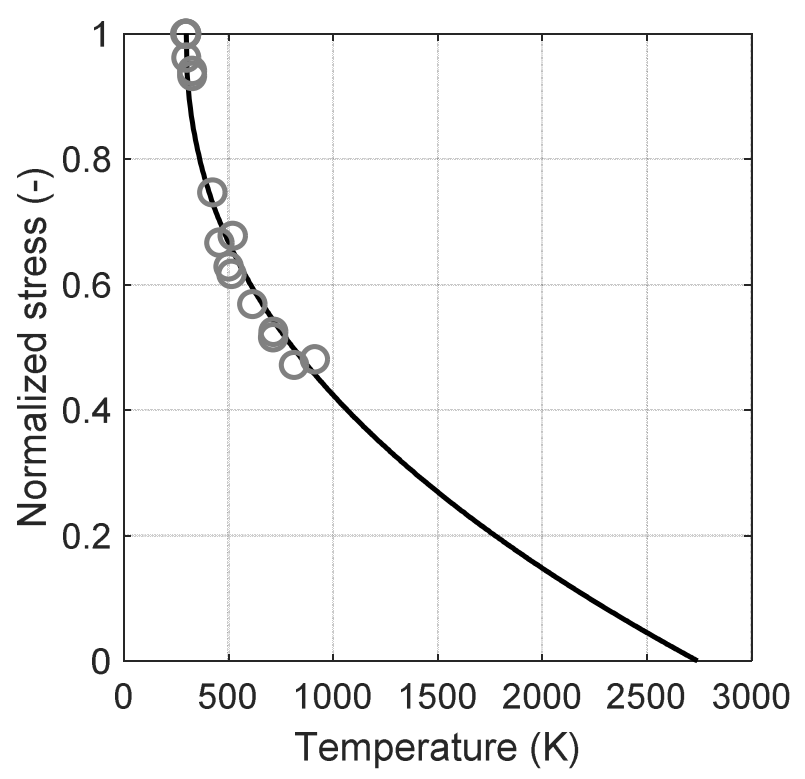

Fig. 7. Thermal softening modelling: the markers represent the data taken from the scientific literature; the solid line represents the fitting model used to build the table $k_{t}$.

Since in the actual testing campaign test at different temperatures were not performed, the authors fit the data available in the scientific literature [5-7] in accordance with the Johnson-Cook formulation. The comparison between the adopted thermal softening model and the thermal data taken from the scientific literature is reported in fig. 7. The aim was to find a proper scale factor to apply to the flow stress vs. effective plastic strain at room temperature (e.g. the room temperature was assumed to be the reference one) to assemble the table $k_{t}$ for different temperature values in the range 
between room temperature and $700{ }^{\circ} \mathrm{C}$ (which is estimated to be consistent with the final temperature in high strain-rate test). The reference curve was assumed equal to the stress-strain curve defined in table $k_{1}$ at the lowest strain-rate.

From a thermal point of view, the quasi-static and low strain-rate simulations were performed as isothermal while the dynamic ones as adiabatic. As widely known in the scientific literature, the problem is related to the intermediate strain-rates, which the authors decided to consider as adiabatic. Because the real behaviour is between the adiabatic and the isothermal conditions, the optimization was performed by considering the experimental data up to necking onset: until this moment the two hypotheses are more or less equivalent since no plastic strain concentration occurs, so the temperature effects are limited.

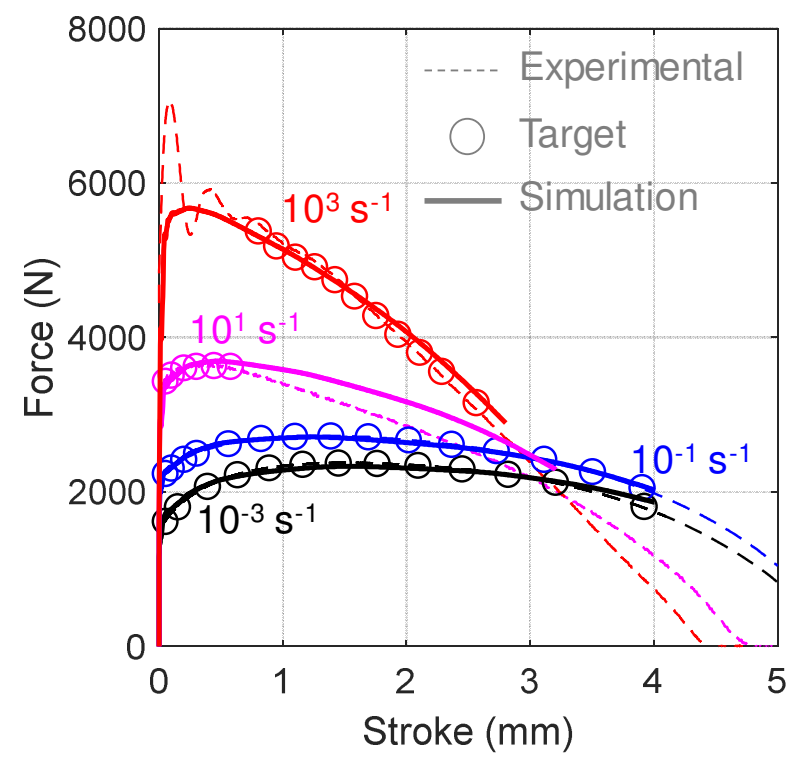

Fig. 8. Comparison between experimental (target) and computed results in terms of force-stroke curves.

As previously discussed, the results of the optimization procedure were 4 different sets of parameters, each of which able to reproduce a particular loading condition. Different cases were tested, by varying the end plastic strain, to limit the maximum level of deformation which produced too high distortion of the FE mesh. The highest value of effective plastic strain for which reliable results were found was about $100 \%$. For this case, the comparison in terms of computed vs. experimental force-stroke curves is reported in fig. 8, where the markers represent the targets points on which the MSE evaluation was performed, the dashed lines the entire experimental curves and the solid lines the computed curves. The initial oscillations at high strainrates are mainly due to inertia (the steel pin used for the sample fixing and the head of the sample have a considerable mass) and are not physically related to the material behaviour, hence they were omitted from the parameters identification procedure. By comparing the curves of fig. 8 , it is possible to conclude that the entire tabular model is able to predict the material response at different loading conditions both in terms of material strength by varying the strain as well as in term of necking onset prediction. This aspect, for example, is very important: as a matter of fact, if the same optimization procedure was used to identify a unique J-C model, it would not be able to predict a variation of the necking onset $(\mathrm{d} \sigma / \mathrm{d} \varepsilon=\sigma)$ induced by a variation of the strain-rate condition, since it is a pure multiplicative model (by neglecting the self-heating contribution in dynamic loading conditions). Similarly, with a unique $\mathrm{J}-\mathrm{C}$ model, it would not be possible to predict a variation of the hardening behaviour by changing the strain-rate. The tabular model can be visualized at a fixed temperature (e.g. the reference one) as a surface which describes the equivalent stress as a function of effective plastic strain and strain-rate, as reported in fig. 9.

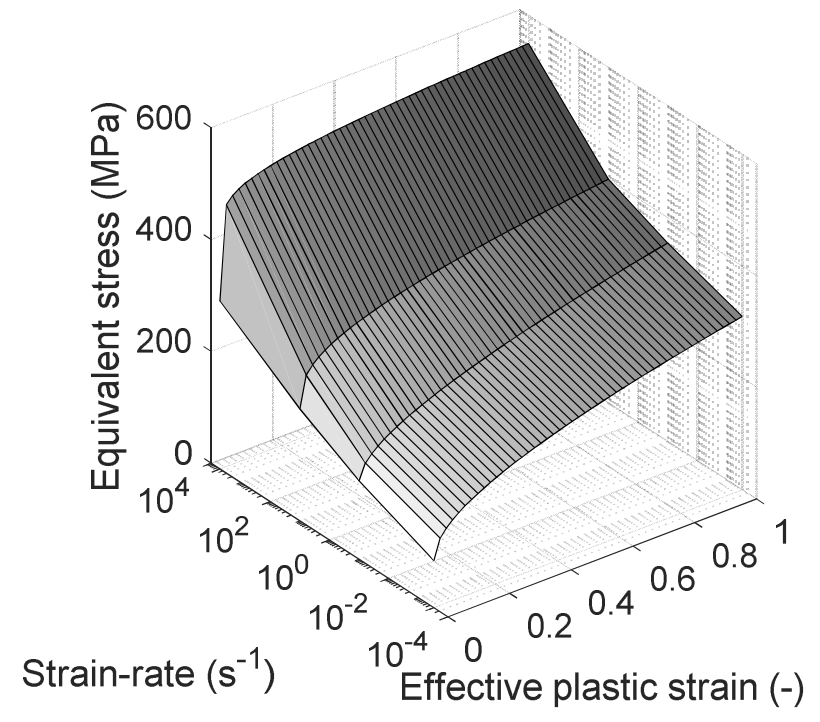

Fig. 9. Surface defining equivalent stress as a function of effective plastic strain and strain-rate defined by the optimized tabular strength model, $T=298 \mathrm{~K}$.

\subsection{Model validation}

The diagram of fig. 8 proved that the optimized flow stress curves were able to reproduce the material response in term of strength, but it did not assure that the solution obtained in terms of strain distribution along the specimen is consistent with the experimental one. This is essentially due to the fact that the necking profile was not taken into account in the optimization procedure. A post-optimization check was performed by directly comparing the experimental vs. computed specimen shapes in the necking region. A qualitative comparison was performed for two cases which correspond to the test nominally performed at $10^{-3} \mathrm{~s}^{-1}$ and $10^{3} \mathrm{~s}^{-1}$ of strain-rate. In fig. 10, the comparison is reported for a maximum effective plastic strain value of $100 \%$. The computed result is overlapped to a half of the correspondent experimental image. In both the cases, the comparison was satisfying: the strength model can predict both the width of the minimum cross-section and the shape of the entire necking zone. The dark shadow 
which can be saw at high level of deformation is not due to an underestimation of the necking cross-section but is the profile of the opposite face and it came from the fact that the machining process produced trapezoidal specimens.

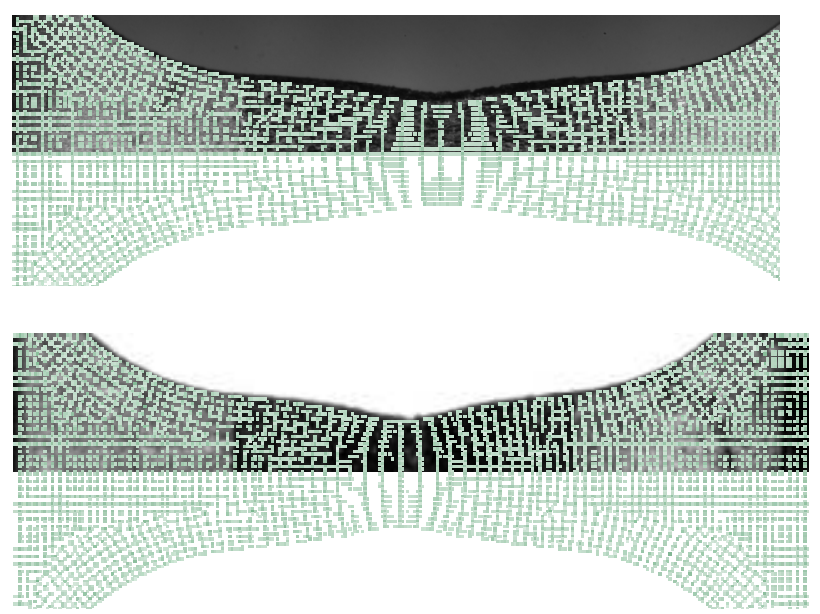

Fig. 10. Necking profile comparison between experimental and computed results for the test nominally at $10^{-3} \mathrm{~s}^{-1}$ and $10^{3} \mathrm{~s}^{-1}$ of strain-rate (maximum effective plastic strain 100\%).

\section{Conclusions}

In this work, an ad-hoc testing campaign was performed to get data on the strain-rate sensitivity in tension of pure sheet niobium. The final objective was the identification of a reliable strength material model to be used in numerical simulations of the forming process adopted to produce RF Crab-Cavity at CERN of Geneva in the framework of the HL-LHC upgrade. The experimental tests were performed at room temperature at 4 different strain-rates by covering 6 orders of magnitude. As expected for a pure BCC metal, the experimental results confirmed the high sensitivity to strain-rate, which strongly influences not only the material strength, but also the necking onset: by increasing the strain-rate, the strain at necking is reduced and the most part of the test is in necking regime. Therefore, the stress state is no longer uniaxial but is becomes strongly triaxial. This implied the use of a reverse engineering approach based on FE simulations to identify the flow stress curve.

A preliminary data analysis suggested that simple models, such as the J-C one, were not able to correctly predict the material response over wide ranges of variation of strain and strain-rate with a unique set of parameters. From this the choice to adopt a tabular strength model (MAT_224 in LS-DYNA) in which different flow stress curves can be defined at different levels of strain-rate and temperature. The final objective of this work was the identification of the curves able to correctly predict the material response both in terms of material strength and necking onset and profile. This was performed via a Multi-Case Optimization, in which the flow stress-strain curves at 4 different strain-rates were identified by using a reverse engineering approach. The strain-rates were chosen in order to include the minimum and the maximum experimental values. The optimization was based on the minimization of the Mean Squared Error between computed and experimental force-stroke curves. To correctly compute the self-heating in adiabatic loading condition, the temperature sensitivity was obtained by processing data taken from the scientific literature. The optimized strength model was able to correctly compute the material behaviour up to $100 \%$ of effective plastic strain. The results of the numerical simulations showed the material model was able to correctly predict the material strength by varying the loading condition as well as the reduction of the strain at instability. Finally, the consistency in terms of necking profile was checked at the end of the optimization process. Even in this case, the comparison allowed concluding that the model accuracy for the material behaviour prediction was very high.

The authors are grateful to the Mechanical and Material Engineering Group (Fabrication Methods \& Subcontracting and Forming \& Welding groups) of CERN (Geneva) for providing the material subject of this study.

\section{References}

1. C. Zanoni, et. al., The Crab Cavities Cryomodule for SPS test, IPAC17, Copenhagen, Denmark (2017)

2. G. Apollinari, I. Bejar Alonso, M. Lamont, M. Rossi, High-Luminosity Large Hadron Collider (HL-HLC): Preliminary Design Report CERN (2015)

3. M. Scapin et al., Int. J. Imp. Eng. 106 (2017)

4. LS-DYNA® Keyword user's manual - Volume II, LS-DYNA R9.0, LSTC (2016)

5. N. Nemat-Nasser, W. Guo, Mater. Sci. Eng. A, 284, 1-2 (2000)

6. G. Z. Voyiadjis, F. H. Abed, Mech. Mater, 37, 2-3 (2005)

7. H. Lim, et al., J. Mech. Ph. Solids, 74 (2015) 